\title{
Solution of the Generalized Bethe-Goldstone Equation for ${ }^{16} \mathrm{O} *$
}

\author{
J. Schlitter \\ Institut für Theoretische Physik II, Ruhr-Universität Bochum \\ (Z. Naturforsch. 28 a, 1586-1590 [1973] ; received 9 July 1973)
}

\begin{abstract}
The generalized Bethe-Goldstone equation from the $e^{\mathrm{s}}$-theory is used to perform a calculation in the harmonic oscillator model. It takes into account hole-hole diagrams and eliminates the model-dependent particle energies. Both effects tend to increase the binding energy and to decrease the nuclear radius.
\end{abstract}

\section{Introduction}

So far perturbation theory has been used most widely as an approach to the many-body problem in nuclear physics, leading to the Goldstone series for observables such as energy and RMS radii. An alternative method, briefly denoted as $e^{\mathrm{S}}$-theory, was introduced by Coester and Kümmel ${ }^{1}$ which start from a suitable ansatz for the many-body wave function. The main advantage of this theory which contains a classification in terms of $n$-body correlations from the beginning is the possibility of treating the expressions by algebraic means. A solution of the ground state problem without degeneracy was given by Kümmel ${ }^{2}$. He described a technique to extract $\mathrm{n}$-body cluster functions from the full wave function and discussed the resulting equations in connection with the well known diagramatic representations.

Here we want to apply Kümmel's generalized Bethe-Goldstone (GBG) equation for correlated twobody functions to the ground state of ${ }^{16} \mathrm{O}$. The differences in comparison with the Bethe-Goldstone (BG) equation are investigated both theoretically and by numerical calculations.

As a shell model (SM) approximation we use the harmonic oscillator $(\mathrm{HO})$ model although an exact calculation should also involve the determination of the single particle functions in a self-consistent way. The problem of self-consistency in connection with the GBG equation is studied separately in the work of Kümmel and Zabolitzky ${ }^{3}$.

\section{The Generalized Bethe-Goldstone Equation}

The A-particle bound state for a fermion system can be written as ${ }^{1}$

$$
|\psi\rangle=\exp \left[\sum_{n=1}^{A} S_{n}\right]|\Phi\rangle
$$

* Thesis, Bochum 1971, abbreviated.

Reprint requests to: Institut für Theoretische Physik II, Ruhr-Universität-Bochum, D-4630 Bochum, Universitätsstraße 150, POB 2148. if one only assumes that $|\psi\rangle$ is orthogonal to the SM determinant

$$
|\Phi\rangle=\prod_{v_{i}}^{A} a_{v_{i}}^{+}|0\rangle \equiv\left|v_{1} \ldots v_{A}\right\rangle
$$

which is used as a starting approximation. The operators

$$
\begin{aligned}
S_{n}= & \left(1 /(n !)^{2}\right) \sum_{\varrho_{1} \ldots \varrho_{n}} \sum_{v_{1} \ldots v_{n}} a_{\varrho_{n}}^{+} \ldots a_{\varrho_{1}}^{+} \\
& \times\left\langle\varrho_{1} \ldots \varrho_{n}\left|S_{n}\right| v_{1} \ldots v_{n}\right\rangle a_{v_{1}} \ldots a_{v_{n}}
\end{aligned}
$$

annihilate $n$ holes (labeled by $v, \mu, \ldots$ ) and create $n$ particles (labeled by $\varrho, \sigma, \ldots$ ) in the subspace of non-occupied states. For $n>1$ they describe n-particle correlations since they cannot be reduced to product terms, or, in the language of graphes, they are represented by "linked diagrams".

Among the two alternative versions concerning the 1-particle part we choose the one where $S_{1}=0$ which is equivalent to the "maximum overlap" condition ${ }^{4}|\langle\psi \mid \Phi\rangle|^{2}=\max$. Thus the rôle of the proper correlations is exhibited and the equations are simplified at the same time.

By inserting the ansatz (1) into the Schrödinger equation

$$
\left[\sum_{i}^{A} T(i)+\sum_{i<j}^{A} V(i j)-E\right]|\psi\rangle=0
$$

where $T(i)$ are the kinetic energies and $V(i j)$ the two-body potentials one can derive coupled equations for the hole state functions $\langle x \mid v\rangle$ and for correlated n-particle functions. Restriction to 2-particle correlations leads to the GBG equation for the correlated 2-particle functions

$$
\begin{aligned}
\left\langle x_{1} x_{2}\left|\psi_{2}\right| v_{1} v_{2}\right\rangle= & \left\langle x_{1} x_{2} \mid v_{1} v_{2}\right\rangle \\
& +\left\langle x_{1} x_{2}\left|S_{2}\right| v_{1} v_{2}\right\rangle,
\end{aligned}
$$

which reads

$$
\begin{aligned}
{\left[T_{x_{1}}\right.} & \left.+T_{x_{2}}+V\left(x_{1} x_{2}\right)\right]\left\langle x_{1} x_{2}\left|\psi_{2}\right| v_{1} v_{2}\right\rangle \\
& +\left[U\left(x_{1}\right)+U\left(x_{2}\right)\right]\left\langle x_{1} x_{2} \mid v_{1} v_{2}\right\rangle \\
& -\left(\varepsilon_{v_{1}}+\varepsilon_{v_{2}}\right)\left\langle x_{1} x_{2}\left|\psi_{2}\right| v_{1} v_{2}\right\rangle \\
& =\left\langle x_{1} x_{2}\left|[1-Q] V \psi_{2}\right| v_{1} v_{2}\right\rangle \\
& -\left\langle x_{1} x_{2}\left|S_{2} \Pi V \psi_{2}\right| v_{1} v_{2}\right\rangle .
\end{aligned}
$$


We have used abbreviations for the projection operators

$Q=\frac{1}{2} \sum_{\varrho \sigma}|\varrho \sigma\rangle\left\langle\varrho \sigma\left|, \quad \Pi=\frac{1}{2} \sum_{v \mu}\right| v \mu\right\rangle\langle v \mu|$,

which project onto the space of excited and occupied 2-particle states, respectively, and for the Hartree-Fock (HF) potential

$U\left(x_{1}\right)\left\langle x_{1} x_{2} \mid \nu_{1} \nu_{2}\right\rangle=\mathscr{A}_{v}\left\langle x_{1}|U| \nu_{1}\right\rangle\left\langle x_{2} \mid \nu_{2}\right\rangle$

where $\mathscr{A}_{v}$ is the antisymmetrization operator with respect to the $v_{i}$.

The states $|v\rangle$ have to diagonalize a HF matrix with eigenvalues $\varepsilon_{v}$

$$
\left\langle v|T| v^{\prime}\right\rangle+\left\langle v|U| v^{\prime}\right\rangle=\delta_{v v^{\prime}} \varepsilon_{v}
$$

where the HF potential satisfies the self-consistency condition

$$
\langle\alpha|U| v\rangle=\sum_{v^{\prime}}\left\langle\alpha v^{\prime}\left|V \psi_{2}\right| \nu v^{\prime}\right\rangle
$$

for arbitrary states $|\alpha\rangle$. Thus the problem of correlations is connected with the determination of the hole states $|v\rangle$ and the hole energies $\varepsilon_{v}$. The total energy is given by

$$
E=\sum_{v}\langle v|T| v\rangle+\frac{1}{2} \sum_{\nu v^{\prime}}\left\langle v v^{\prime}\left|V \psi_{2}\right| v v^{\prime}\right\rangle .
$$

The GBG equation is most easily discussed in connection with the ordinary BG equation if it is reduced to an equation for $S_{2}$ alone by subtracting the 1-particle equation. For excited states the theory does not contain any prescription because they never occur as individual states. We define, however, a potential $U^{\prime}$ such that the equation

$$
\left[T+U^{\prime}-\varepsilon_{Q_{i}}\right]\left|\varrho_{i}\right\rangle=0
$$

yields an orthonormalized basis $\left|\varrho_{i}\right\rangle$ in the subspace of excited states. $U^{\prime}$ cannot be choosen to be zero except for the special case of nuclear matter. Then we find for $S_{2}$ the equation

$\left\langle\varrho_{1} \varrho_{2}\left|S_{2}\right| \nu_{1} \nu_{2}\right\rangle=\frac{1}{\varepsilon_{\varrho_{1}}+\varepsilon_{\varrho_{2}}-\varepsilon_{v_{1}}-\varepsilon_{\nu_{2}}}$

$\cdot\left\{-\left\langle\varrho_{1} \varrho_{2}|V| v_{1} v_{2}\right\rangle-\left\langle\varrho_{1} \varrho_{2}\left|V S_{2}\right| v_{1} \nu_{2}\right\rangle\right.$

$-\left\langle\varrho_{1} \varrho_{2}\left|S_{2} \Pi V\right| v_{1} v_{2}\right\rangle-\left\langle\varrho_{1} \varrho_{2}\left|S_{2} \Pi V S_{2}\right| v_{1} v_{2}\right\rangle$

$$
\left.+\left\langle\varrho_{1} \varrho_{2}\left|\left[U_{1}{ }^{\prime}+U_{2}{ }^{\prime}\right] S_{2}\right| v_{1} v_{2}\right\rangle\right\}
$$

which is represented graphically in Figure 1.

Formal solution of the integral equation by iteration shows that part A alone produces the well known ladder diagrams from Brueckner theory, so

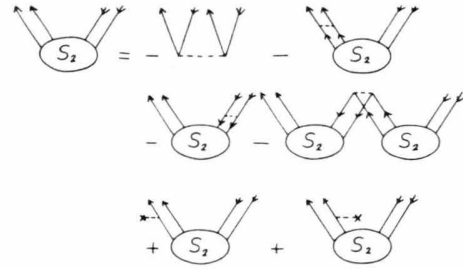

Fig. 1. Graphical representation of Eq. (12) for $S_{2}$.

that the BG equation is contained in the generalized equation.

Part B, however, leads to down and upgoing ladders not included therein. It describes intermediate scattering into the Fermi sea, but whithout introducing hole amplitudes into the wave function.

A modified BG equation was also given by Galitski ${ }^{5}$ and Iwamoto ${ }^{6}$ to take into account diagrams of this kind. Their equation, however, does not avoid hole amplitudes and leads to energy denominators which are no more the difference of particle and hole energies.

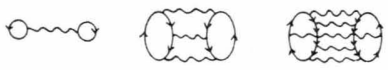

Fig. 2. Some energy diagrams summed up by solving the GBG equation.

Figure 2 shows diagrams for the potential energy which are summed up in a closed form by solving the GBG equation. They are expressed here by the Brueckner matrix $G$ containing all graphs with upgoing ladders.

Part C corrects for the fact that the particle energies in the denominator contain potential energies for excited states. These are, of course, as arbitrary as is the choice of excited states, so they must be compensated by the 1-particle potentials inserted in particle lines. It can be seen from the diagrams $\mathrm{C}$, however, that matrix elements $\left\langle v\left|U^{\prime}\right| \varrho\right\rangle$ must vanish due to the orthogonality condition

$$
\left\langle\mu_{1} \mu_{2}\left|S_{2}\right| v_{1} \nu_{2}\right\rangle=0 \text {. }
$$

This is not satisfied in general by model potentials which are used to replace $U$ and $U^{\prime}$, but it may not be crucial for the $\mathrm{HO}$ potential which is almost diagonal.

\section{Treatment in the Harmonic Oscillator Model}

In order to study the influence of the new terms we have performed a calculation for ${ }^{16} \mathrm{O}$ in the $\mathrm{HO}$ model following mainly the method of Kallio and 
Day ${ }^{7}$. Self-consistency has been taken into account approximately (a) by shifting the hole energies and the diagonal matrix elements of $U$ so that Eqs. (8) and (9) were satisfied, and (b) by determining the oscillator parameter $\hbar \omega$ from the condition for nondiagonal matrixelements

$\sum_{\alpha \nu}\left|\langle\alpha|U(\hbar \omega)| v\rangle-\sum_{v^{\prime}}\left\langle\alpha v^{\prime}\left|V \psi_{2}\right| v v^{\prime}\right\rangle\right|^{2}=\min$.

As usual the operators $\psi_{2}$ and $S_{2}$ have been restricted to act only on the relative part of the 2-particle wave functions. This leads to a set of integro-differential equations which may be coupled for relative triplet states if the tensor force can admix a component with a relative angular momentum different from the original one. Note that the projector $\Pi$ introduces an additional coupling among different center-of-mass (CM) states of the 2-particle system.

The use of a shell model requires corrections concerning the CM motion of the whole nucleus. For the kinetic energy $\left\langle T_{\mathrm{CM}}\right\rangle$ and the sqare radius $\left\langle R_{\mathrm{CM}}^{2}\right\rangle$ we have taken the exspectation values given by the uncorrelated model functions and subtracted them to obtain the binding energy $\mathrm{BE}$ and the RMS radius. The Coulomb corrections for the binding energy are taken from Eden, Emery, and Sam$\operatorname{panthar}^{8}$.

\section{Results and Discussion}

We have solved numerically 3 versions of the 2-particle equation: (A) is the usual BG equation, (B) includes hole-hole diagrams, and (C) is the full GBG equation including also the compensation of particle energies. The notation corresponds to the one used above, version $\mathrm{B}$ contains the terms $\mathrm{A}$ and $B$, etc. The calculations have been performed for the Hamada-Johnston potential ${ }^{9}$ which has a repulsive hard core, and the Reid soft core potential ${ }^{10}$.

The relative wave functions (Fig. 3 a) are not very much altered by the additional terms taken into account. In the figure their influence can be seen only in the defect functions (Fig. $3 \mathrm{~b}$ ) which are the difference of the correlated and the uncorrelated functions. They exhibit stronger oscillation over the hole range, particularly for $(\mathrm{C})$, but the short-range character of the correlations is conserved. As mentioned above the orthogonality $\left\langle v_{1} v_{2}\left|S_{2}\right| v_{1} v_{2}\right\rangle=0$ cannot be satisfied completely for $(\mathrm{C})$ because of the
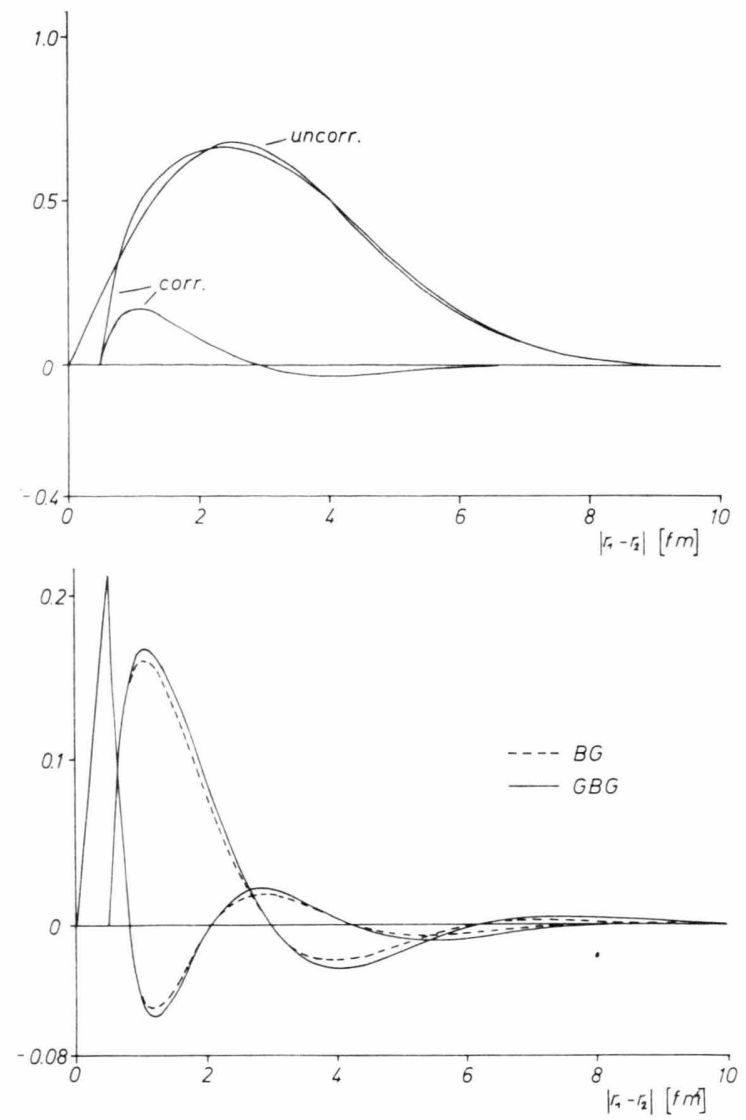

Fig. 3. Relative functions in the ${ }^{3} \mathrm{~S}_{1}$ state for the lowest $\mathrm{CM}$ state calculated with the Hamada-Johnston potential. (a) shows the correlated and uncorrelated wave functions including the small $l=2$ component. In (b) the defect functions are drawn as they result from the $\mathrm{BG}$ and the GBG equation.

model potential used. For (A) we find an overlap of $10^{-3}$, whereas for $(\mathrm{C})$ we obtain a value of $10^{-2}$, which means that orthogonality is essentialy conserved.

The behaviour of the wave functions is reflected by the behaviour of the diagonal relative $t$-matrix elements, $t=V \psi_{2}$, which determine the energy. Their absolute values as well as the potential energy are increased if the same oscillator parameter is used (Table 1). Both corrections with respect to the original BG equation lead to greater binding energy. The main effect of 0.8 to $0.9 \mathrm{MeV}$ comes from the lack of particle energies in our BGB equation. The hole-hole diagrams summed up by solution of the intermediate version (B) yield only $0.2 \mathrm{MeV}$. This is true over the whole range of oscillator parameters $\hbar \omega$ for which we have calculated the energies ((Figs. $4 \mathrm{a}, \mathrm{b})$. 
Table 1. Energies and corrected binding energies for $\hbar \omega=13 \mathrm{MeV}$.

\begin{tabular}{llrrrrrr}
\hline \multicolumn{9}{c}{ Hamada-Johnston } \\
\hline version & A & B & C & A & \multicolumn{1}{c}{ B } & C & \\
E/A & -1.96 & -2.16 & -3.08 & -3.19 & -3.27 & -4.07 & $\mathrm{MeV}$ \\
BE/A & 1.73 & 1.93 & 2.85 & 2.96 & 3.04 & 3.84 & $\mathrm{MeV}$ \\
\hline
\end{tabular}
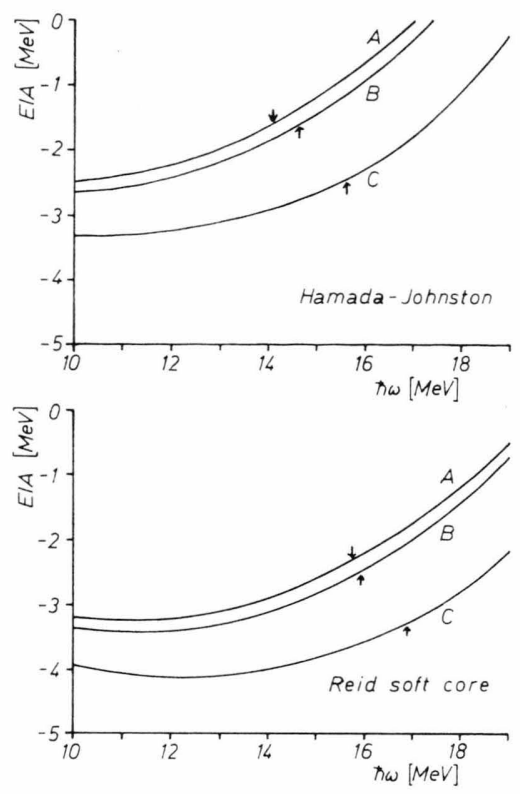

Fig. 4. Energy per nucleon E/A for different versions of the 2-particle equation as a function of the oscillator parameter $\hbar \omega$ without CM and Coulomb corrections. The arrows mark the self-consistent values.

It is clear, however, that one should compare the results not at a fixed value $\hbar \omega$, but for those values where the approximate self-consistency condition (13) is satisfied (Table 2). Then it turns out that the net effect becomes smaller because greater values of $\hbar \omega$ are needed to achieve self-consistency. This points to the important rôle the HF problem is playing in the theory of correlations for finite nuclei. Note that self-consistency is not satisfied for a maximum of binding energy as function of $\hbar \omega$. This has no physical meaning as it is not connected with a variational principle.
It is well known ${ }^{11}$ that the RMS radius is mainly determined by the model parameters and is only little decreased by short range correlations. We have found that for a fixed oscillator parameter it is not significantly affected by the small changes of the wave functions if we pass from the $B G$ to the BGB equation. It is shifted to smaller values only due to the self-consistency prescription for $\hbar \omega$.

The comparison of calculated values for energy and radius with the experiment is still unsatisfactory. Both the inclusion of hole-hole diagrams and the elimination of the model particle energies tend to increase the binding energy, but one is far from reaching the experimental value of $7.98 \mathrm{MeV}$. The best value $3 \mathrm{MeV}$ is given by the Reid soft core potential. While the energy is improved the radius moves away from the experimental value $2.57 \mathrm{fm}$ up to $2.27 \mathrm{fm}$.

From the investigation of phase shift equivalent potentials 12 it is known that the discrepancy between theory and experiment cannot be removed by choosing other 2-particle potentials considered. This is in agreement with our results. On the other hand, we have seen that the effects considered here are closely related to the self-consistency problem. Therefore it seems reasonable to solve first the complete Brueckner-Hartree-Fock problem for finite nuclei before higher correlations are taken into account.

The author should like to thank Professor Dr. H. Kümmel as well as Dr. K. H. Lührmann and Dr. J. G. Zabolitzky for many helpful discussions.

Table 2 . Bindung energies and RMS radii at self-consistent values $\hbar \omega$.

\begin{tabular}{cccccccc}
\hline \multicolumn{1}{c}{ Hamada-Johnston } & \multicolumn{7}{c}{ Reid soft core } \\
\hline version & $\mathrm{A}$ & $\mathrm{B}$ & $\mathrm{C}$ & $\mathrm{A}$ & $\mathrm{B}$ & $\mathrm{C}$ & $\mathrm{MeV}$ \\
$\hbar \omega$ & 14.1 & 14.6 & 15.6 & 15.7 & 15.9 & 16.8 & $\mathrm{MeV}$ \\
$\mathrm{BE} / \mathrm{A}$ & 1.4 & 1.4 & 2.1 & 2.2 & 2.3 & 3.0 & $\mathrm{MeV}$ \\
$\left\langle r^{2}\right\rangle 1 / 2$ & 2.46 & 2.42 & 2.33 & 2.35 & 2.33 & $2.27 \mathrm{fm}$ & \\
\hline
\end{tabular}


1 F. Coester, Nucl. Phys. 7, 421 [1958].

H. Kümmel, Lectures on the Many-Body Problem, ed. E. R. Cajanello, Academic Press, New York 1962.

2 H. Kümmel, Nucl. Phys. A 176, 205 [1971].

3 H. Kümmel and J. G. Zabolitzky, Phys. Rev. C 7, 547 [1973].

4 L. Schäfer and H. A. Weidenmüller, Nucl. Phys. A 174, 1 [1971].

5 B. M. Galitski, J. Exp. Theor. Phys. UDSSR 7, 104 [1958].

6 F. Iwamoto, Progr. Theor. Phys. (Kyoto) 22, 903 [1959].
7 A. Kallio and B. D. Day, Nucl. Phys. A 124, 177 [1969].

8 R. J. Eden, V. J. Emery, and S. Sampanthar, Proc. Roy. Soc. London A 253, 177 [1959].

9 T. Hamada and I. D. Johnston, Nucl. Phys. 34, 383 [1962].

10 A. Reid, Ann. of Phys. 50, 411 [1968].

11 M. Fink, H. Hebach, and H. Kümmel, Phys. Rev. C 3, 2075 [1971].

12 F. Coester, S. Cohen, B. D. Day, and C. M. Vincent, Phys. Rev. C 1, 769 [1970]. 